\title{
Supersolid phases in the extended boson hubbard model
}

\author{
Kwai-Kong Ng and Y. C. Chen \\ Department of Physics, Tunghai University, Taichung, Taiwan
}

(Dated: August 17, 2021)

\begin{abstract}
We present a comprehensive numerical study on the ground state phase diagram of the twodimensional hardcore boson extended Hubbard model with nearest $\left(V_{1}\right)$ and next nearest neighbor $\left(V_{2}\right)$ repulsions. In addition to the quantum solid and superfluid phases, we report the existence of striped supersolid and three-quarter (quarter) filled supersolid at commensurate density $\rho=0.75$ (0.25) due to the interplay of $V_{1}$ and $V_{2}$ interactions. The nature of three-quarter filled supersolid and the associated quantum solid will be discussed. Quantum phase transition between the two supersolids of different symmetries is observed and is clearly of first order.
\end{abstract}

PACS numbers: 75.10.Jm, 05.30.Jp, 75.40.Mg

Supersolid (SS) state [1, 2], on which both diagonal and off-diagonal long-range order are broken, has been intensively discussed recently on various models $[3,4$, 5, 6, 7, 8]. This is partially due to the experimental advance of optical lattice that one day may investigate those hardcore boson models and the exotic supersolid phase experimentally. Furthermore, supersolid phase of spin models is also of great interests as these quantum spin systems has been suggested could be realized in real materials $[\underline{6}, 7,8]$.

The simplest hardcore boson model that includes only the nearest neighbor (nn) interaction, however, does not stabilize the supersolid phase on square lattice [3, 9]. To induce the supersolid phase, one may relax the hardcore constraint to softcore [4] or to include the next nearest neighbor (nnn) interactions [3]. For the latter case, a striped supersolid (SS1) phase is found associated with the half-filled striped solid (QS1) phase, where bosons form stripes that break the $x-y$ symmetry. This stripe structure allows the superfluid component to easily flow through the channels between stripes and therefore coexistence of both solid and superfluid ordering is possible. Unlike striped solid, the hardcore checkerboard solid provides no pathway for superfluid component and no checkerboard SS of hardcore boson has been found so far, unless nnn hopping is included [10]. In this work, we present a comprehensive study on the phase diagram of hardcore boson hubbard model with nn and nnn interactions. A three-quarter filled supersolid that, like the checkerboard SS, preserves the $x-y$ symmetry is found stabilized in a large parameter regime of $V_{1}$ and $V_{2}$. Associated to the supersolid is a three-quarter filled quantum solid, which share the same star-like occupation pattern (see inset of Fig 11). For clarify, we call this solid and supersolid as the star solid (QS2) and star supersolid (SS2) hereafter. Interestingly, the supersolids, SS1 and SS2, that possess different underlying symmetries compete in some parameter regimes in which, as we will show, first order phase transition occurs, in contrast to recent work on a similar model [10]. We tackle the problem with both quantum Monte Carlo (QMC) and variational
Monte Carlo (VMC) methods which give consistent result. A generic Jastrow wave function in $\mathrm{VMC}$ is able to generate qualitative features of all phases in QMC calculations.

We study the extended boson Hubbard model on a 2D square lattice with the Hamiltonian

$$
H=-t \sum_{i, j}^{n n}\left(b_{i}^{\dagger} b_{j}+b_{i} b_{j}^{\dagger}\right)+V_{1} \sum_{i, j}^{n n} n_{i} n_{j}+V_{2} \sum_{i, j}^{n n n} n_{i} n_{j}-\mu \sum_{i} n_{i}
$$

where $b\left(b^{\dagger}\right)$ is the boson destruction (creation) operator and $\sum^{n n}\left(\sum^{n n n}\right)$ sums over the (next) nearest neighboring sites. To set the energy scale of the problem, we let $t=1$ throughout the paper. At half filling, the ground state can be a checkerboard solid (with wave vector $(\pi, \pi)$ ) for strong $\mathrm{nn}$ coupling $V_{1}$, or a striped solid (with wave vector $(\pi, 0)$ or $(0, \pi))$ for strong nnn coupling $\left.V_{2} \quad 3\right]$. For competing values of $V_{1}$ and $V_{2}$, however, quantum frustration disfavors both solid structure and leads to the condensation of bosons instead, i.e. a superfluid ground state. Upon doping for large $V_{2}$, as mentioned above, striped solid structure provides channels of superflow so that extra bosons can form superfluid on top of the striped structure and leads to a striped supersolid. Note that quantum effect eventually drives all bosons to participate the superflow, although the superfluidity transverses to the stripes is much smaller [3]. For dominating $V_{1}$, on the other hand, addition bosons forms no condensate on the checkerboard solid because domain formation is energetically more favorable. As a result, no supersolid of checkerboard solid ordering is found. The phase diagram of half filling and result of doping close to half filling have been discussed in detail in reference [3].

Remarkably, when further increasing doping to $\rho=$ 0.75 , our numerical calculations show a rich phase diagram that contains a superfluid (SF) phase, a star solid phase which have finite structure factor $S(\mathbf{Q}) / N=$ $\sum_{i j}\left\langle n_{i} n_{j} e^{i \mathbf{Q r}_{i j}}\right\rangle / N^{2}$ at $\mathbf{Q}_{0}=(\pi, \pi),=(\pi, 0)$, and $=(0, \pi)$, and supersolid phases of either star ordering or striped ordering. The result obtained from QMC calculation with the stochastic series expansion (SSE) algorithm [11] on 


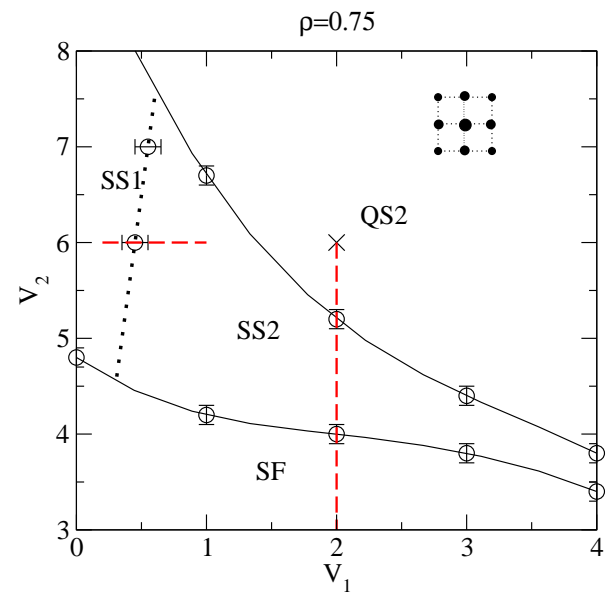

FIG. 1: (color online) Ground state phase diagram of $V_{2}$ as a function of $V_{1}$ for density $\rho=0.75$. First (second) order phase transition is denoted by dotted (solid) lines. The inset shows the boson occupation profile (the star pattern) of the QS2 and SS2 phase. The cross shows a representative point of QS2 phase at $\rho=0.75$ where the order parameters are plotted in Fig. 2 Lattice size of $36 \times 36$ and $28 \times 28$ are used with temperature $\beta=1 / 2 L$.

square lattice is presented in Fig 1 In QMC, the superfluidity, given by $\rho_{s}=\left\langle W^{2}\right\rangle / 4 \beta t$ is computed by measuring the winding number fluctuation. The calculation is done on scanning over different $\mu$ grand canonically to search for the right $\mu$ that fix the density $\rho=0.75$ for each coordinates $\left(V_{1}, V_{2}\right)$ in the diagram. Due to particle hole symmetry of the Hamiltonian $H$, one must obtain the same phase diagram as in the Fig 1 for $\rho=0.25$. Hereafter we will focus on $\rho=0.75$ but all discussion applies to $\rho=0.25$ as well. Let us now discuss each phases in more detail, starting with the star solid QS2.

Inset of Fig 1 shows the ordering of the QS2. The lattice contains four square sublattices with twice of the lattice constant. It is important to stress that QS2 is not a solid with three sublattices fully occupied and the fourth totally empty which naturally give $\rho=0.75$. Our calculation shows that all sites have finite occupations as shown in the figure. As shown, two sublattices are identical because of the $x-y$ symmetry. A typical structure of QS2 has one of the sublattice almost fully occupied and the two identical sublattices have occupation $n$ with the last sublattice has occupation $\sim 2(1-n)$. At $V_{1}=2.0$ and $V_{2}=6.0$, the occupations on different sublattices are $0.99,0.37,0.37$ and 0.27 respectively. It is not surprising that quantum fluctuation and the gain in kinetic energy favor this ordering than the ordering with one sublattice being empty. One important feature of the QS2 phase is that, although the $S\left(\mathbf{Q}_{0}\right) / N$ is finite, it is rather small compare to those of striped solid QS1. In Fig, 2 , we show the order parameters as a function of $\mu$ with $V_{1}=2$ and $V_{2}=6$, a representative point (the cross in Fig 1) of SS2 phase in the phase diagram, from which we obtain all

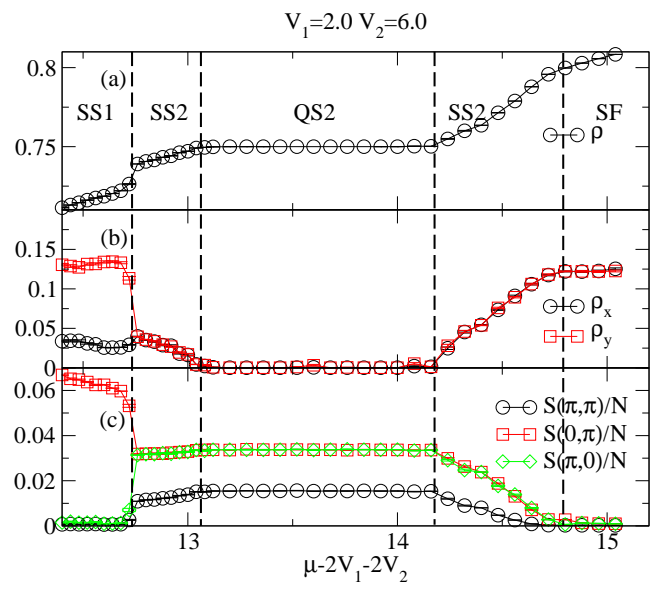

FIG. 2: (color online) QMC result of (a) boson density $\rho$, (b) superfluidity $\rho_{x}$ and $\rho_{y}$, and (c) structure factor of wavevectors $(\pi, \pi),(0, \pi)$ and $(\pi, 0)$ as functions of chemical potential $\mu-2 V_{1}-2 V_{2} . V_{1}=2.0, V_{2}=6.0$ and lattice size is $28 \times 28$.

different phases by varying $\mu$.

A clear plateau appeared in the density curve signals the existence of a solid phase, in which the superfluid density $\rho_{x(y)}$ vanishes but $S\left(\mathbf{Q}_{0}\right) / N$ (Fig 22) is finite and remains flat throughout the QS2 phase. Structure factor of all other wavevectors are essentially zero but $S\left(\mathbf{Q}_{0}\right) / N$ remains finite under finite size analysis. It is note that the striped solid at half filling has $S(\pi, 0) / N \sim 0.2$ (not shown). The small value of $S\left(\mathbf{Q}_{0}\right) / N$ in the QS2, therefore, indicates the solid QS2 is rather soft.

Away from the density $\rho=0.75$ there are supersolid phases (SS2), as shown in Fig 2, which is characterized by the same star solid ordering (wavevector $\mathbf{Q}_{0}$ ) as the QS2 state and appears on both increasing or reducing $\mu$ away from the QS2 phase. In this phase $\mathrm{x}-\mathrm{y}$ symmetry is preserved such that $S(\pi, 0) / N=S(0, \pi) / N$ and is about twice of $S(\pi, \pi)$. All these peaks reduces simultaneously away from the QS2 and vanish at the same critical point where SF emerges at larger $\mu$. This implies that this SS2 is a unique phase characterized by these wavevectors but not a mixture of striped phase. Remarkably, like the QS2 state, SS2 has one of the sublattices is almost fully occupied and does not participate on the superflow. Therefore the superfluid flows only on the other three sublattices. This self-pinning effect of one sublattice is rather rare and may worth further investigation [12]. Transition from QS2 to SS2 is of second order as both $\rho_{x(y)}$ and $S\left(\mathbf{Q}_{0}\right) / N$ changes continuously across phase boundaries and no abrupt change in order parameters is observed.

On the other hand, there is clearly a first order phase transition from SS2 to SS1 as shown in Fig 2 where all parameters exhibit a sudden jump at the $\mu-2 V_{1}-2 V_{2} \approx$ 12.72. This discontinuous transition arises from the distinct broken crystal symmetry of the two supersolids. Our VMC calculation, presented later, also supports the 


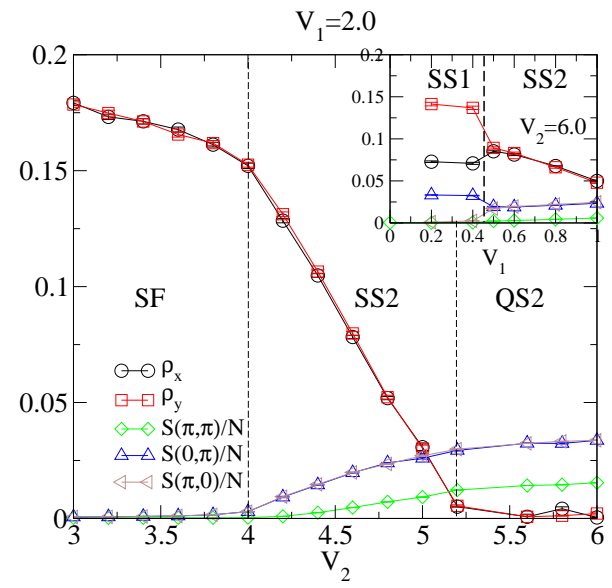

FIG. 3: (color online) Ground state order parameters of a $28 \times 28$ lattice at $\rho=0.75$ for different $V_{2}$. Inset shows the first order phase transition at $V_{2}=6.0$.

discontinuous phase transition. It is worthy noting that a recent study on the same model but with nnn hoping $t^{\prime}$ included also observes the QS2 and SS2 phases by Chen et al. [10]. Our result indicates that $t^{\prime}$ plays no significant role on the stabilization of both QS2 and SS2 phases which, indeed is a direct consequence of competition between $V_{1}$ and $V_{2}$ interactions. Furthermore, contrary to our findings, Chen et al. observe a crossover from SS1 to SS2. Whether it arises from $t^{\prime}$ is still unclear yet.

Moving down from the cross along the dotted line in Fig. 1, the width of the QS2 plateau shrinks as $V_{2}$ is reduced. When $V_{2} \approx 5.2$ the QS2 phase disappears and the ground state changes continuously to the SS2. Order parameters as functions of $V_{2}$ are shown in the Fig. 3 with $V_{1}=2$ (dotted line in Fig.11). By reducing the $\mathrm{nnn}$ repulsion, the system gains kinetic energy that favors superfluidity and soften the solid structure at the same time. Consequently, QS2 continuously changes to SS2 and to SF eventually at $V_{2}=4.0$ where all peaks of $S\left(\mathbf{Q}_{\mathbf{0}}\right) / \mathbf{N}$ vanish simultaneously. Note that there is a large parameter range where SS2 is stabilized at this commensurate density $\rho=0.75$.

More complicated situation arises for $V_{1}<1$ where the $\mathrm{nn}$ repulsion is too weak to support the star-like structure against the striped one. Fig. 1 shows the emergence of SS1 at small $V_{1}<1$ within the SS2 regime. The phase transition between SS1 and SS2 is again of first order because of the different broken translational symmetries (inset of Fig (3). For vanishing $V_{1}$, neither SS2 and QS2 stabilized so that only ground states of striped ordering is found for all fillings, consistent to the previous findings [3]. In other words, the necessary condition for the appearance of the star-like quantum solid and supersolid is the competition between nn and nnn interactions.

To further investigate the effect of finite $V_{1}$, we plot in Fig. 4 the phase diagram of fixed $V_{1}=2$ with varying

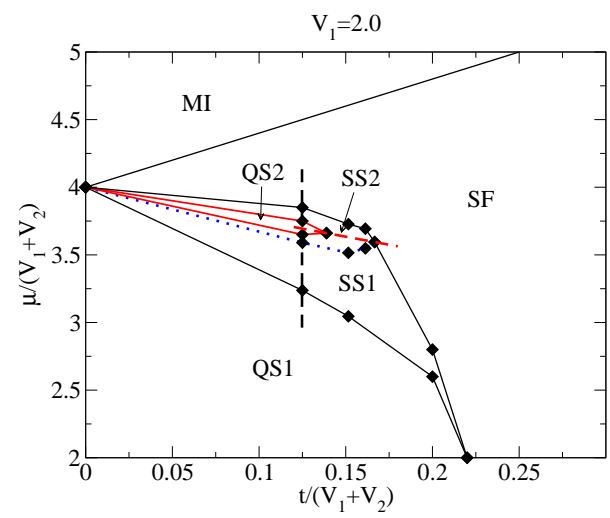

FIG. 4: (color online) Phase diagram of $\mu$ vs $t$ for fixed $V_{1}=$ 2.0. The lines are guide to the eyes with dashed line (solid line) stands for first (second) order phase transitions. The change of order parameters along the black dotted line at $V_{2}=6.0$ is plotted in Fig. 2 while those along the red dotted at fixed $\rho=0.75$ is displayed in Fig. 3 .

$\mu$. The phase diagram is similar to the case of vanishing $V_{1}$ (see ref.[3]) except there are two new phases, SS2 and QS2, emerges within the phase of SS1. Within this phase, increasing $\mu$ (e.g. along the dashed line) such that $\rho$ approaches $3 / 4$, the star-like ordering becomes energetically more favorable than the striped ordering as discussed before and SS2 or QS2 is stabilized. Note that this happens only when $V_{2}>4$, otherwise the SS1 phase simply dissolves into SF phase upon increasing $\rho$. The emergence of SS2, and QS2 in the phase diagram reflects the interplay of nn and nnn interaction in the system.

Now let us present our results by VMC in support of the star solid QS2 and SS2 found in QMC calculation. The wave function we used is the standard Jastrow wave function which is defined as :

$$
|\Psi\rangle=e^{-\sum_{i \neq j} v_{i, j} n_{i} n_{j}}\left|\Phi_{0}\right\rangle
$$

where $\left|\Phi_{0}\right\rangle=\left(b_{k=0}^{\dagger}\right)^{N}|0\rangle$ is the non-interacting superfluid wave function and $N$ is the total number of bosons. In order to incorporate all kinds of phases in the same wave function, the pair-wise potential $v_{i, j}$ 's are independently optimized by the algorithm proposed by Sorella [13]. To determine the phase diagram, we calculate the number of bosons in the zero momentum mode, the condensate, $N_{k=0}=b_{k=0}^{\dagger} b_{k=0}, S(\pi, 0)$ and $S(\pi, \pi)$ for the optimized wave function. In Fig. 5 (a) three order parameters are shown as a function of density for given $V_{1}=4$ and $V_{2}=6$. As density increases, the phase changes from $\mathrm{SF}$ to SS2 around the QS2 at $\rho=0.25$. With $\rho>0.28$ $S(\pi, 0)$ vanishes and the system becomes in SS1 phase and QS1 at $\rho=0.5$. This is consistent with the phase diagram (Fig. 1) obtained by QMC. In order to verify if the new SS2 is thermodynamically stable, we show the boson density as a function of the $\mu$ which is calculated from the energy of adding add a particle to the system 


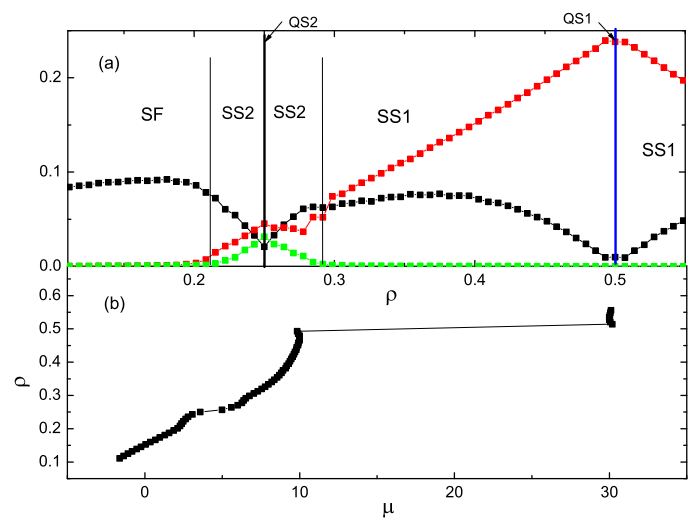

FIG. 5: (color online) VMC results of (a) the condensate $N_{k=0}$ (square), structure factor of wavevectors $(\pi, \pi)$ (circle) and $(0, \pi)$ (triangle) as functions of density and (b) boson density $\rho$ as a function of the chemical potential. Here $V_{1}=4$, $V_{2}=6$ and lattice size is $24 \times 24$.

$\mu=E(N+1)-E(N)$. Two plateaux are found at density $\rho=0.25$ and 0.5 which correspond to QS2 and QS1, respectively. Positive slope around the plateau at $\rho=0.25$ manifests that the SS2 found is stable against phase separation. Although there are discrepancies on the position of the phase boundaries, the Jastrow wave function alone captures the essential features and successfully generates all the observed phases in QMC.

In Fig. 6(a) we show the order parameters as a function of $V_{2}$ with $V_{1}=4$ and $\rho=0.25$. The three phases found in Fig. 3 for $V_{2}=2$ are also observed here. The representative optimized variational parameters $v\left(r_{i j}\right)$ for $\mathrm{SF}\left(V_{2}=2\right), \operatorname{SS} 2\left(V_{2}=5.5\right)$ and $\mathrm{QS} 2\left(V_{2}=8\right)$ are shown in Fig. 6(b). The data $(r \geq 2)$ is fitted to an exponential form $A e^{-r / \xi}$ with decay length $\xi=2.0,2.21$ and 2.68 for $\mathrm{SF}, \mathrm{SS} 2$ and QS2 respectively. The large value of $v\left(r_{i j}\right)$ and $\xi$ in QS2 phase indicates the existence of a strong long range repulsion between bosons while the interaction is shorter ranged in SS2 and SF phases accordingly.

In summary, we present numerical evidences for the appearance of a quantum solid, a supersolid phase with star pattern and a striped supersolid at or around $\rho=0.75$ or 0.25 . The competition between $\mathrm{nn}$ and $\mathrm{nnn}$ interaction is found to be important for observation of both QS2 and SS2. A detail study is given on the ground state phase diagrams by varying $V_{1}$ and $V_{2}$ as well as the chemical potential $\mu$. The quantum phase transition between SS1 and SS2 is appeared to be first order because of the abrupt change of translational symmetry. Our VMC calculation also support the QMC findings and the simple Jastrow wave function alone is adequate to generate all the phases consistent with the QMC calculations.

The authors thank M.F. Yang for fruitful discussions and acknowledge the support of the National Center for
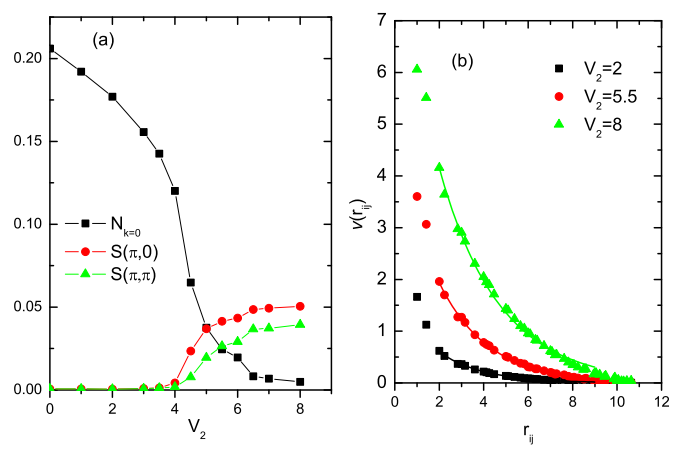

FIG. 6: (color online) (a) VMC results of the condensate $N_{k=0}$, structure factor of wavevectors $(\pi, \pi)$ and $(0, \pi)$ as functions of $V_{2}$ with $V_{1}=4$ and the boson density $\mathrm{n}=0.25$. The definition of the symbols are the same as Fig. 5. (b) The variational parameters $v_{i, j}$ vs. $r_{i j}$ for $V_{2}=2,5.5$ and 8 . The solid lines are the fitting functions of $A e^{-\xi r_{i j}}$. The lattice size is $24 \times 24$.

Theoretical Science. K.K.N. acknowledges financial support by the NSC (R.O.C.), grant no. NSC 95-2112-M029-010-MY2 and NSC 95-2110-M-029-004.YCC is supported by NSC 95-2112-M-029-003-MY3. Part of the calculation is supported by the National Center of High Performance Calculation(Taiwan).

[1] O. Penrose and L. Onsager, Phys. Rev. 104, 576 (1956).

[2] A.F. Andreev and I.M. Lifshitz, Soviet Physics JETP 29, 1107 (1969); G.V. Chester, Phys. Rev. A, 2, 256 (1970); A.J. Leggett, Phys. Rev. Lett. 25, 1543 (1970).

[3] G.G. Batrouni and R.T. Scalettar, Phys. Rev. Lett. 84, 1599 (2000); F. Hebert, et al.. Phys. Rev. B 65, 014513 (2001).

[4] P. Sengupta, L. P. Pryadko, F. Alet, M. Troyer, and G. Schmid, Phys. Rev. Lett. 94, 207202 (2005).

[5] S. Wessel and M. Troyer, Phys. Rev. Lett. 95, 127205 (2005); D. Heidarian and K. Damle, ibid 95, 127206 (2005); R.G. Melko et al., ibid 95, 127207 (2005).

[6] K.K. Ng and T.K. Lee, Phys. Rev. Lett. 97, 127204 (2006).

[7] N. Laflorencie and F. Mila, Phys. Rev. Lett. 99, 027202 (2007).

[8] P. Sengupta and C. Batista, Phys. Rev. Lett. 98, 227201 (2007).

[9] G. Schmid, S. Todo, M. Troyer, and A. Dorneich, Phys. Rev. Lett. 88, 167208 (2002).

[10] Y.C. Chen, R. Melko, S. Wessel, and Y.J. Kao, cond-mat 0708.1807

[11] A.W. Sandvik, Phys. Rev. B 59, R14157 (1999); ibid 56, 11678 (1997); O.F. Syljuåsen and A.W. Sandvik, Phys. Rev. E 66, 046701 (2002).

[12] K.K. Ng, in preparation.

[13] M. Capello, F. Becca, M. Fabrizio, and S. Sorella, Phys. Rev. Lett. 99, 056402 (2007); S. Sorella, Phys. Rev. B 
71, 241103 (2005). 